\title{
Capacity Analysis for Equity Funds
}

\author{
Michael O'Neill, Camille Schmidt, \\ AND GEOFFREY WARREN
}

Michael O’Neill

is a portfolio manager for Investors Mutual, Ltd. in Sydney, Australia, and an adjunct associate professor at Bond University in Gold Coast, Australia. michael.oneill@iml.com.au

\section{Camille Schmidt}

is a market insights manager at SuperRatings in Sydney, Australia. camille.schmidt@superratings .com.au

\section{GeOFFrey WARREN}

is an associate professor at the Australian National University in Canberra, Australia. geoff.warren@anu.edu.au
$\mathrm{W}$ e address the estimation of capacity for an equity fund that forms portfolios based on a given investment strategy. We initially refine how capacity is defined as effective capacity, which is the amount of assets under management (AUM) at which a fund becomes unable to make further active investments that generate alpha $^{1}$ at the margin in excess of a minimum threshold. We then outline and illustrate an approach for estimating effective capacity that combines the main determinants in an integrated analysis. The key drivers of capacity are identified as being the number of opportunities available to the fund, the market segments from which opportunities are sourced, the alpha profile, the cost of executing trades, and (importantly) any constraints on stock holdings or ability to participate in trades. Combining these drivers into a unified analysis offers two advantages. First, it allows for interactions. For example, capacity may be determined by the combined impact of the rate of alpha accrual, execution costs (ECs), and trade participation constraints, with the latter potentially acting to prevent the capture of alpha before it erodes to below the cost of trading. Second, an integrated analysis accommodates the fact that the factors that limit capacity differ across strategies. For instance, capacity for longerhorizon strategies such as value investing tends to be determined by the number of opportunities in combination with holding constraints. Meanwhile, constraints on the ability to trade quickly and on the cost of trading are most relevant for shorter-horizon strategies such as momentum investing. The approach we present allows the key determinants of capacity to emerge for each strategy.

We highlight issues that arise when undertaking capacity analysis in practice. We note how a fund may become unable to invest further in opportunities that add value at the margin for investors, even though on average substantial alpha is being generated from existing active investments. This possibility has important implications for how capacity is defined, analyzed, and interpreted. Many equity portfolios comprise a combination of active alpha-seeking positions and a core component that is implicitly beta exposure, perhaps reflecting holdings to limit tracking error. We propose that effective capacity be defined and analyzed with respect to the alpha-seeking component only, focusing on the marginal alpha generated from additional investment rather than overall fund alpha. Other important considerations include the scope and nature of available opportunities, overlap with other investors trading on similar strategies, and accounting for the potential drivers of capacity not captured by the analysis. 
This article is arranged as follows. We initially provide background on existing research into the evaluation of capacity and then discuss its definition. Our integrated approach for capacity analysis is then outlined and subsequently illustrated for two strategies. We end with concluding comments.

\section{BACKGROUND}

Three strands of research relate to the analysis of capacity: theoretical models of optimal trading or portfolio construction under alpha erosion and trade frictions, empirical estimates of capacity for specific equity strategies, and capacity analysis undertaken within the investment management industry. The theoretical research has its genesis in the implementation shortfall (IS) approach of Perold [1988]. At the foundation of IS is the concept that the alpha available under an investment strategy can be dissipated by either the EC incurred upon trading or the opportunity cost (OC) arising from either deferral or failure to trade. The link between IS and capacity arises because greater AUM implies larger trade sizes, which in turn leads to either increased EC and/or impaired ability to trade in volume before alpha erodes. Eventually IS erodes alpha below a minimum acceptable level at some AUM, which in turn establishes capacity. This dynamic was first discussed by Perold and Salomon [1991].

The literature building on the work of Perold [1988] initially focused on identifying optimal trading strategies that minimize IS (e.g., Arnott and Wagner [1990], Bertsimas and Lo [1998], Almgren and Chriss [2001], Almgren et al. [2005], and Huberman and Stanzl [2005]). More recently, authors have addressed the implications of costly trading and alpha decay for optimal portfolio construction, including Kahn and Shaffer [2005]; Coppejans and Madhavan [2007]; Engle and Ferstenberg [2007]; Serbin, Bull, and Zhu [2009]; Amihud and Mendelson [2013]; and Gârleanu and Pedersen [2013]. Applying these models to capacity analysis is a natural step, but it has only been considered by some of these authors. The complexity of the problem and the preference for closed form solutions has led to stylized formulations. ${ }^{2}$

Empirical research has mainly focused on estimating capacity for selected market anomalies, ${ }^{3}$ including work by Korajczyk and Sadka [2004]; Chen, Stanzl, and Watanabe [2005]; Vangelisti [2006]; Frazzini, Israel, and Moskowitz [2012]; Landier, Simon, and Thesmar [2015]; and
Novy-Marx and Velikov [2016]. There is considerable variation in the sample, method, and findings across these studies for similar strategies. ${ }^{4}$ Notable points of difference in method include the estimation of ECs, assumptions about portfolio construction, constraints imposed, allowance for trading discretion, and how capacity is defined.

Finally, most of the analysis undertaken in the investment industry is not publicly available. We queried a range of players on their approach to capacity analysis and encountered a wide range of methods, indicating no consensus, coupled with a propensity to rely on simpler measures that are easy to calculate.

In sum, there appears to be no clear agreement on how capacity analysis should be undertaken. We aim to help address this situation by proposing a workable definition, scoping the range of methods for evaluating capacity, and suggesting a practical approach that captures the key drivers in an integrated analysis.

\section{DEFINING CAPACITY}

The literature provides disparate definitions of capacity. Our definition modifies the definitions outlined by Vangelisti [2006]:

Effective capacity: The amount of AUM invested in an active strategy at which it is no longer possible to make additional investments that generate marginal alpha in excess of a minimum threshold.

Our definition is closest to wealth-maximizing capacity, which is the AUM that maximizes the product of alpha and AUM. However, it also incorporates the notion of a minimum required alpha threshold, hence integrating the wealth-maximizing and threshold definitions. ${ }^{5}$ This definition focuses on alpha generation at the margin, identifying capacity as the AUM after which a fund earns alpha below a minimum acceptable level on an additional dollar invested. The alpha threshold represents the minimum alpha required by investors to cover the management fee and compensate for active risk. This threshold may vary with the nature of the fund investors, including their alpha requirements and the fee they pay, or across market segments (e.g., a higher alpha threshold might be imposed on small stocks in recognition of greater research costs or higher risk). A key implication of this definition is that average alpha may sit well above the threshold, at the point at which opportunities to earn marginal alpha exceeding that threshold are exhausted. In contrast, a threshold 
capacity definition implies that it is acceptable for a fund to continue investing until average alpha is diluted to the threshold, even though additional investment may not be adding value. Furthermore, the wealth-maximizing definition implies continued investment, providing that marginal alpha is positive. For instance, if the alpha threshold is $2 \%$, effective capacity occurs where the marginal dollar invested earns less than $2 \%$, rather than zero as implied under wealth-maximizing capacity.

Focusing on the alpha-seeking component of a portfolio is important. Many funds combine alpha and beta, with the latter manifesting as core positions perhaps designed to control tracking error. Because core beta exposure is generally available at a large scale for minimal cost, capacity analysis is relevant only with respect to the alpha-seeking component. Once capacity for the active component is identified, it can be recombined with any beta component for analysis of the total fund and the appropriate fee.

Two issues arise in relation to the scope of the strategy being evaluated. The first is the boundary of the strategy itself - specifically, how far a fund may adjust its investment process to mitigate the adverse effects of increasing AUM. We recommend that capacity analysis rule out changes to the underlying investment signal on which positions are based, while allowing for the possibility that a fund could adjust how it trades and forms portfolios based on the signal, including expanding the breadth of opportunities to which the signal is applied. The second issue relates to the boundaries of AUM investing on a strategy. A fund may be pursuing a similar strategy and hence sharing capacity with other funds within the same organization or other investors in the market. The overlap is often partial, but it is relevant because it blurs the lines and makes it harder to identify capacity with precision. We suggest formally accounting for overlapping positions within an investment organization, recognizing that aspects such as holding and trading constraints operate at the organizational rather than fund level. This might be supplemented by subjectively evaluating the effect of other investors using similar strategies.

\section{CAPACITY ANALYSIS:}

\section{AN INTEGRATED APPROACH}

Four methods for undertaking capacity analysis for equity funds are outlined by O'Neill and Warren [2016] and illustrated by O'Neill, Schmidt, and Warren [2016]:
1. Rules of thumb: Notional limits are placed on portfolio metrics considered symptomatic of capacity, such as AUM as a percentage of market capitalization in the market segment being addressed, or average time to exit a portfolio.

2. Ex post analysis: The portfolio is monitored for indications of diminishing ability to implement a strategy as AUM increases via tracking selected portfolio metrics, including number of positions; measures of benchmark convergence, such as tracking error or active share (Cremers and Petajisto [2009]); post-trade analysis of time to trade and EC; IS (Perold [1988]); and alpha trends.

3. Simulation analysis: A portfolio or its observed trades are scaled up with the aim of gauging capacity through evaluating the impact of greater AUM on expected alpha or the percentage of holdings either becoming subject to ownership constraints or exceeding a limit on time-to-trade.

4. Predictive models: This entails applying theoretical models of the type described in the "Background" section. Frazzini, Israel, and Moskowitz [2012] and Landier, Simon, and Thesmar [2015] provide examples.

Our approach sits in the predictive model category. The key advantage of predictive models is that they can incorporate various influences on capacity into an integrated analysis, potentially including the expected alpha profile arising from a strategy, transaction costs, constraints on trading and stock holdings, and allowance for optimal implementation of investment signals. Our approach aligns with the class of predictive models that focuses on locating the optimal trade schedule for a given target or paper portfolio, which amounts to minimizing IS. It thus draws on the principles outlined by Perold [1988] and the intuitions underpinning research on optimal trade scheduling (e.g. Almgren and Chriss [2001]).

Our aim is to form a parsimonious model that encapsulates the key drivers of capacity, yet remains sufficiently basic to be applied by industry practitioners. To do so, we do not allow for optimal portfolio construction or transaction cost risk. Models incorporating these aspects resort to stylized solutions to solve the multiperiod dynamic optimization problem. For instance, Gârleanu and Pedersen [2013] achieved a closed form solution by assuming that stock returns follow a 
factor-driven process with constant fade, whereas Almgren and Chriss [2001] observed that incorporating risk into problems of optimal execution comes at the cost of needing to assume prices follow a random walk. By eschewing these features, we gain functionality in other ways that are important for the analysis of capacity. In particular, our approach has the flexibility to accommodate alpha accrual profiles of any timing and shape and is capable of readily incorporating constraints on trade volumes and stock holdings.

Our approach involves identifying the opportunities available to generate alpha; profiling the expected alpha arising from those opportunities; and then optimizing alpha capture subject to ECs, holding constraints, and trading constraints. The model minimizes IS for the investment signal under an active strategy via optimizing the trade schedule by trading off EC and OC. The model is run at varying levels of AUM, generating expected net alpha as a function of AUM. Effective capacity is identified as the AUM at which it is no longer possible to make further investments that earn marginal alpha net of EC in excess of the threshold.

\section{Key Variables}

We now list and discuss the main variables incorporated in the model:

- Number of opportunities: The number of opportunities to generate alpha under a strategy is a crucial variable. Holding all other factors constant, a doubling in opportunities would double the AUM that can be productively invested, implying a doubling of capacity. In practice, the relation is more complex because additional opportunities are often available only at lower alpha. We address this issue by modeling access to second-best opportunities as a further round of investment once preferred opportunities are exhausted.

- Market segments from which opportunities are sourced: Capacity is likely to be lower when alpha opportunities are sourced from smaller or less-liquid stocks, simply because the AUM that can be successfully deployed to capture alpha is more limited. Our approach envisages segmenting the portfolio, estimating capacity for each segment, and then aggregating.
- Alpha profile: Both the magnitude and timing of alpha generation are relevant. Higher gross alpha creates a larger buffer for more AUM to be deployed before net alpha after EC is eroded to the threshold. The timing of alpha accrual can dictate the factors that are most important for capacity. When alpha accrues over a shorter horizon-such as in momentum or short-term reversal strategiesthe ability to invest AUM quickly without alpha being negated by EC tends to be critical. When the alpha accrual is delayed and extended - such as in fundamental value investing - the ability to trade quickly is often of little consequence. Rather, the number of opportunities and holding constraints tend to be critical for capacity. Alpha profiles may be estimated by analyzing historical returns arising from signals to invest under the strategy.

- Cost of executing trades: As EC cuts into the buffer between gross alpha and the alpha threshold, its relevance for capacity depends on its significance relative to the difference between gross alpha and the alpha threshold. EC reflects aspects such as required turnover and the segment from which opportunities are sourced. It is estimated using a transaction cost function.

- Holding constraints: Limits on the percentage of market capitalization that may be held act as a hard constraint that strictly limits capacity; once binding, it is no longer possible to deploy further AUM. Holding constraints can prove a critical driver when alpha accrues over long horizons or is sourced from smaller stocks.

- Trade participation constraints: The main impact of trade participation constraints is to force deferral of trades as AUM increases. This becomes influential when it is important to invest before residual alpha is eroded. Trade constraints are hence more relevant for strategies of shorter duration.

- Trade scheduling: This involves optimizing the trade schedule, with deferral of trades indicated when the EC savings from reducing trade size exceed the OC from trade delay. Our analysis suggests that gains from trade scheduling are modest unless the EC cost function is much steeper than the OC function, and permanent price impacts are small. Trade optimization often tends to have only a marginal impact on alpha and rarely has a meaningful impact on capacity itself. 


\section{Steps in the Analysis}

Our approach involves eight steps, some entailing simplifying assumptions:

1. Identify the opportunities to generate alpha: Historical analysis is conducted to identify points at which changes in portfolio positions were signaled under the strategy. For an actual fund, ideally the decision point should be identified, although the first trade leading to a significant portfolio change may suffice. For a strategy based on formal signals, such as factor scores, the opportunities might be represented by portfolios formed periodically from the top-ranked stocks.

2. Segment the sample: The extent to which the sample should be segmented for analysis depends on whether the key inputs vary across the sample in a manner that would make broad averages misleading. Division into size segments is natural. Other possibilities include liquidity groupings or industry sectors.

3. Profile alpha arising from the signal: A representative alpha profile for each segment is generated by creating a cumulative alpha index ${ }^{6}$ with respect to each position change and computing an average. The alpha profile defines the maximum potential alpha arising under the strategy, prior to IS.

4. Specify portfolio construction rules: Three quantities characterize how the alpha signal is captured in the context of a dynamically managed portfolio. These combine to determine the frequency and size of position changes:

a. Number of stocks: The total number of stocks held reflects the breadth of opportunities generated under the strategy.

b. Rebalancing horizon: The period over which positions are held should relate to the alpha profile, with rebalancing ideally occurring at the point that alpha accrual ends or tapers off. Attention might also be paid to the actual holding period for active positions because funds may not always exit at the optimal point. There is direct correspondence between the rebalancing horizon and turnover and thus EC; for example, a sixmonth rebalancing horizon equates to turnover of $200 \%$ per annum for the active component. ${ }^{7}$ c. Rebalancing interval: This should reflect the frequency at which opportunities arrive. The concept is that the portfolio is notionally divided into a series of rolling slices with one rebalanced at the end of an interval. For instance, a strategy with a six-month horizon and monthly rebalancing would rebalance one-sixth of the portfolio per month for its $200 \%$ turnover.

5. Specify constraints: We include two constraints. The first is the holding constraint, expressed as a limit on the percentage of market capitalization that can be held, with $5 \%$ being a typical choice. The second is the trade participation constraint. A higher trade constraint allows more latitude for the model to determine whether it is worthwhile to participate in the available volume at a greater rate given the associated increased EC; a lower trade constraint will tend to force the deferral of trades as AUM grows. A fund might take advice from its dealing desk on the appropriate assumption. We use 30\%.

6. Specify a transaction cost function: This is used to estimate EC as a function of trade size, with the latter measured as a percentage of average value traded over the time unit of analysis. ${ }^{8}$ The model should capture all costs associated with trading and include both temporary and permanent price impacts. The latter are accumulated over time, such that trades that occur later in the sequence incur the cumulative effect of permanent price impacts.

7. Solve model for a rebalancing of each segment, inputting progressively higher AUM until effective capacity is attained: Solving the model involves identifying the schedule of trades that maximizes net alpha after EC, subject to the constraints. AUM is progressively increased until effective capacity is attained, potentially based around differing alpha thresholds across segments, if so desired. Capacity emerges as a consequence of greater AUM increasing the average position size and hence the required trade size, eventually leading to a situation in which it is no longer possible to make further investments earning marginal alpha in excess of the threshold. This may occur for a number of reasons, including the following: the holding constraint becomes binding for available opportunities; additional trades do not generate marginal alpha in excess of the threshold due to increased EC; and/or the trade participation constraint is encountered, delaying 
implementation to later periods in which the marginal alpha available over the remaining alpha horizon is below the threshold. Although it is not essential to progressively increase AUM to locate capacity, the advantage in doing so is that series are generated for net alpha, IS, EC, and OC, and the manner in which constraints become binding is made apparent. Calculations can be undertaken in a spreadsheet package such as Microsoft Excel, perhaps with the Solver Add-In helping to locate the optimal trade schedule.

8. Accumulate segment results: Estimates of capacity for each segment are summed to estimate overall capacity for the active strategy. Estimates of alpha along with IS and its components for each rebalancing may be annualized, and a weighted sum across the total active portfolio estimated if desired. For funds comprising a combination of alpha and beta, the alpha estimates may be blended with a beta component to form estimates at the total fund level.

The process of analysis not only generates capacity estimates for an active strategy but also provides insight into the critical determinants of capacity for that strategy. The approach also supports undertaking sensitivity analysis around the input assumptions, providing further insight into the critical factors.

\section{Issues to Consider}

The assumptions related to portfolio construction and rebalancing can be influential for the net alpha generated under the model. In practice, a fund may fail to deliver the alpha indicated as a result of suboptimally exiting positions, performance drag associated with nonalpha-seeking positions, or other costs. Whether this invalidates the capacity estimate depends on the extent to which these factors would cause a breach of the alpha threshold at a lower AUM.

Another consideration is the assumptions about the funding side. We conduct our analysis by assuming that buys are funded by sells that incur the same EC and generate zero expected alpha. Hence, we are effectively modeling the process of ongoing rebalancing for a long-only strategy that sells at the end of the alpha horizon and redeploys the funds into stocks at the commencement of their alpha cycle. It may be necessary to model the funding side in some circumstances (e.g., for long-short strategies).

Another issue is that capacity estimates are imprecise and may vary through time. Any model is subject to model and estimation risk, and some key variables can fluctuate with market conditions - for example, the number and nature of opportunities to generate alpha, market liquidity, and EC. The modeling approach supports estimating capacity on the assumption that the historical alpha profile will be repeated going forward under average market conditions. Estimates need to be interpreted carefully, with consideration given to confidence levels and sensitivity to different market conditions. The model provides a broad indication of capacity and the range within which it might sit, along with insight into the most important factors determining capacity for the strategy being examined.

Finally, the capacity of a fund may be affected by other factors, such as economies of scale or scope arising from larger size, diseconomies related to diversion of fund manager focus or coordination problems within investment organizations, and the impact of other investors trading on similar strategies. ${ }^{9}$ The influence of overlapping strategies within an investment organization might be taken into account by allocating the estimated capacity across multiple funds. A more subjective evaluation is probably required in relation to other market participants.

\section{ILLUSTRATING THE APPROACH}

We apply the approach to two strategies in the Australian equity market: a factor-based signal using six-month price momentum and the active positions of a medium-sized value manager, Investors Mutual Limited (IML). These strategies contrast along key dimensions. First, the momentum strategy is based on a quantitative alpha signal, whereas IML is a fundamental stock picker that runs overlapping long-only funds comprising both alpha and beta components. Second, considerable differences in the alpha profiles allow us to illustrate how the nature and timing of alpha generation dictates the critical determinants of capacity. Australia offers the advantage of being a medium-sized market in which ease of investing soon becomes strained upon moving down the list of stocks. This provides a better laboratory to demonstrate capacity analysis than a large 


\section{E X H I B I T 1 Alpha Profiles}

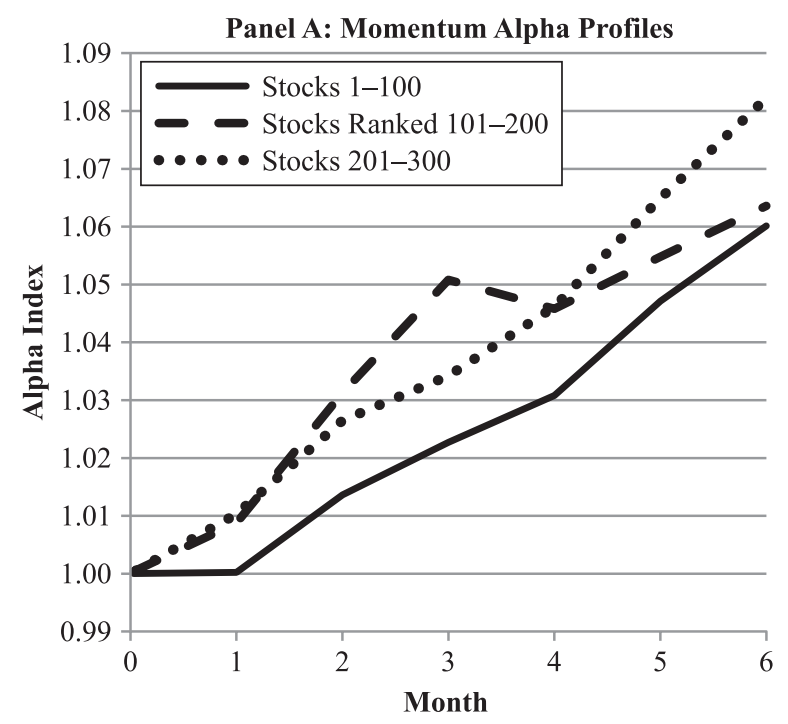

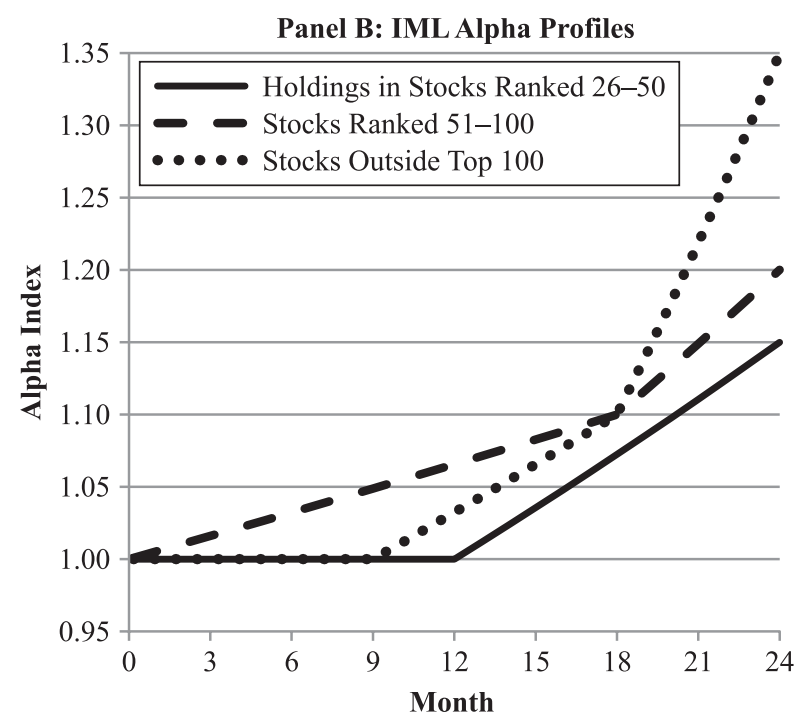

market such as the United States, with its considerable breadth and depth of larger, liquid stocks.

Steps one through three under the approach involve identifying the historic alpha opportunities, segmenting the portfolio, and then generating alpha profiles. For the momentum strategy, we sorted the largest 300 stocks based on total returns over the prior 26 weeks, skipping one week, at the last week in each quarter from September 1997 to June 2016. We then tracked cumulative alpha, measured as total return versus the $\mathrm{S} \& \mathrm{P} /$ ASX300 Index, on a weekly basis. After considering various segmentations and portfolio sorting rules, we settled on dividing the sample into three size segments: large (top 100), medium (next 101-200), and small (next 201-300). Our preferred opportunities are represented by a portfolio of the top 10 stocks in each segment, held over a six-month period. The resulting alpha profiles are plotted in Panel A of Exhibit 1. We also calculated the alpha profile for stocks ranked $11-20$ to test the impact of broadening investment to include second-best opportunities.

For the IML funds, we identified 77 overweight positions, or active bets, taken within their flagship IML Australian Share Fund between January 2002 and June 2016. We then created alpha indexes by tracing returns relative to the S\&P/ASX300 Index. Analysis revealed that IML generates its alpha predominantly from stocks outside of the top 25, with the largest 25 stocks apparently playing the role of core (beta) holdings. We allocated the active alpha-seeking component into three size segments of stocks ranked $26-50$ by market capitalization, stocks ranked 51-100, and stocks outside of the top 100. An alpha accrual horizon of 24 months was adopted, and alpha profiles were smoothed. The series are plotted in Panel B of Exhibit 1. As well as a longer alpha accrual horizon, a key point of contrast is that alpha generation for IML is modest over the first 9-12 months, whereas strong alpha accrual occurs immediately for the momentum strategy. Examining the IML portfolio indicated that it could be characterized as comprising 50\% core beta exposure and a 50\% alpha component composed of about $10 \%$ in stocks ranked $26-50$ and $20 \%$ each in stocks ranked 51-100 and 101-300.

Step four involves specifying the portfolio construction rules. Exhibit 2 presents these rules and what they imply in terms of turnover and average trade size. It also reports the annualized values for the expected alphas over the rebalancing horizon, providing a sense of the relative profitability of the two strategies. Exhibit 2 reveals that turnover for the momentum strategy is four times that for the IML active component, reflecting differences in the alpha accrual and hence rebalancing horizon. However, the momentum strategy has access to more opportunities to generate alpha and is assumed to support 60 stock holdings with 20 in each size segment. IML is assumed to hold 36 active positions, distributed as 


\section{E X H I B I T 2 \\ Portfolio Construction Rules and Annualized Alpha}

\begin{tabular}{lcc}
\hline Strategy & Momentum Strategy & IML Active Positions \\
\hline Definition & $\begin{array}{c}\text { 6-month price } \\
\text { momentum, based } \\
\text { on data 1997-2016 }\end{array}$ & $\begin{array}{c}\text { Payoffs from } \\
\text { observed positions, } \\
\text { 2002-2016 }\end{array}$ \\
Portfolio Construction (active component) & & \\
Alpha accrual and rebalancing horizon & 6 months & 24 months \\
Turnover, per annum & $200 \%$ & $50 \%$ \\
Rebalancing interval & Monthly & Quarterly \\
$\%$ traded at each rebalancing & $16.67 \%$ & $12.5 \%$ \\
Total stocks held across all segments & 60 & 36 \\
Stocks traded per rebalancing & 10 & 4.5 \\
Required trade size as \% of portfolio & $1.667 \%$ & $3.125 \%$ \\
Note: Active component as \% total portfolio & $100 \%$ & $50 \%$ \\
Annualized Expected Alpha & & \\
Preferred opportunities (segment) & & $7.2 \%$ \\
Largest stocks (1-100 and 26-50) & $12.0 \%$ & $9.5 \%$ \\
Medium stocks (101-200 and 51-100) & $12.7 \%$ & $16.2 \%$ \\
Smallest stocks (201-300 and 101-plus) & $16.4 \%$ & $11.7 \%$ \\
Sample average & $13.7 \%$ & n.a. \\
Second best opportunities (ranked 11-20) & & n.a. \\
Largest stocks (1-100) & $2.7 \%$ & n.a. \\
Medium stocks (101-200) & $6.3 \%$ & n. \\
Smallest stocks (201-300) & $9.1 \%$ & \\
Sample average & $6.0 \%$ & \\
\end{tabular}

8 stocks ranked 26-50, 12 ranked 51-100, and 16 outside the top 100 . We also investigate the momentum strategy having access to additional opportunities via stocks ranked 11-20, whereas IML opportunities are treated as strictly limited. The combination of the assumed turnover, rebalancing frequency, and number of stocks results in an average trade size under the momentum strategy of $1.667 \%$ of the portfolio, a bit over half of the $3.125 \%$ for the IML active portfolio. Finally, the alpha profiles indicate that alpha decreases with larger stock size segments under both strategies, the momentum strategy generates slightly greater gross annualized alpha, and alpha drops off sharply for the second-best momentum opportunities.

Step five involves specifying the constraints. We set a baseline holding constraint of $5 \%$ of market capitalization and a trade participation constraint of $30 \%$.

The transaction cost model is addressed in the sixth step. We use the J.P. Morgan market impact model, which estimates EC as a function of trade size, volatility, and bid-ask spreads using Equations (1) and (2):

$$
\begin{gathered}
M I=b * I * \frac{2 \cdot P_{0} V}{1+P_{0} V}+(1-b) \cdot I+\frac{S}{2} \\
I=a 1 \cdot P_{0} V^{a 2} \cdot V_{o} l^{a 3}
\end{gathered}
$$

where $M I$ is the market impact (bps); PoV is the percentage of value traded; $S$ is the spread (bps); $V o l$ is the stock volatility per annum; $b$ is the percentage temporary market impact; $a 1$ is the scaling parameter; $a 2$ is the sensitivity to PoV; and $a 3$ is the sensitivity to volatility.

We combine the J.P. Morgan parameter estimates for Australia in June 2016 with the average values for the input variables for each size segment also estimated in June 2016 and add a notional brokerage cost of 10 bps. Exhibit 3 plots the resulting EC functions on a monthly basis, which is the unit of time used in applying the model. Panel A presents the components based on average inputs for the top-300 stock universe. Panel B plots the EC function for the three size segments examined under the momentum strategy. 


\section{E X H I B I T 3}

\section{Execution Cost (EC) Based on J.P. Morgan Model}
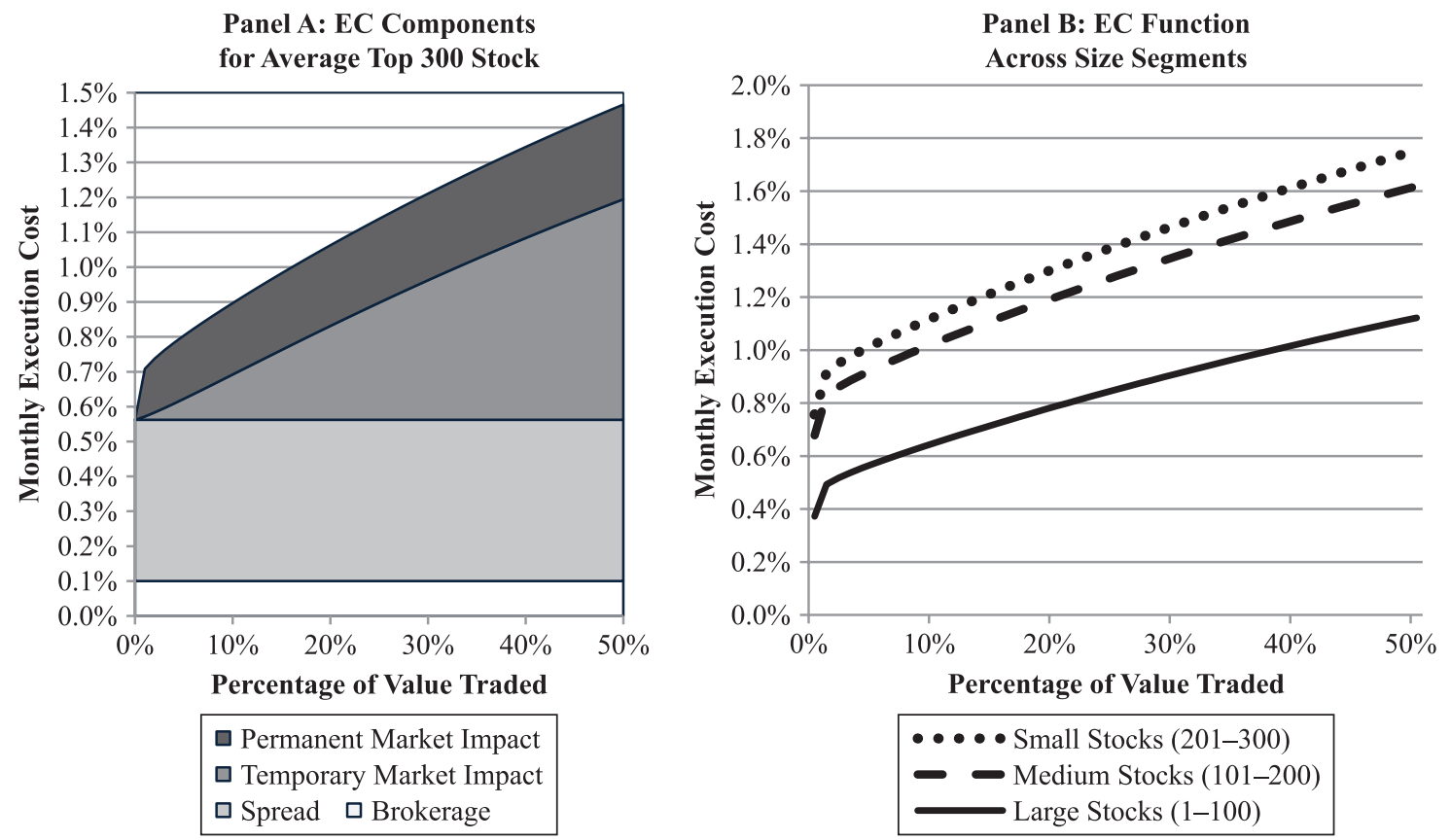

The final steps involve applying the model and generating the results. Specifically, we conduct analysis for an average stock traded for each segment over a rebalancing cycle, and the output is accumulated to the segment and portfolio level. We collect capacity estimates and annualized alpha for each segment, then estimate active strategy totals by aggregating across the segments. We also report the key constraining factor at the point at which effective capacity is identified.

Exhibit 4 plots estimates at various levels of AUM. The top two charts plot expected alpha net of EC for each segment. ${ }^{10}$ The alpha plots reflect average net alpha, with the lines ending at the AUM where marginal net alpha falls to the threshold, hence indicating that effective capacity has been reached. For example, when the momentum strategy reaches effective capacity at $\$ 10.7$ billion in the large stock segment, average alpha is in excess of $6 \%$ and marginal net alpha is $2 \%$. Panels C and D plot the EC and OC components of IS for the medium stock segments.

A number of takeaways emerge. First, expected alpha at effective capacity sits well in excess of the $2 \%$ threshold in all instances. Second, capacity is greater for larger stock segments. Third, the momentum strategy incurs substantially higher alpha erosion due to IS than IML. One reason is its considerably higher turnover, resulting in larger annualized EC. Another reason is that alpha accrual occurs immediately and over a shorter horizon for momentum, making it necessary to trade quickly to capture the alpha. Increases in AUM result in the trade participation constraint becoming binding, which defers trading thus leading to marginal alpha falling below the threshold due to increasing OC. Under the momentum strategy, the greater IS for small and medium stocks results in lower net alpha than for large stocks, notwithstanding a higher expected alpha prior to implementation. For IML, its slow burn alpha profile results in minimal alpha loss from deferral of trades, which manifests in low OC. Furthermore, IML's alpha profile supports rescheduling trades to limit IS, whereas the momentum strategy benefits from trading quickly, making deferring trades of little value. Such features emerge naturally from our integrated modeling approach.

Exhibit 5 reports baseline capacity and alpha estimates, as well as estimates under alternative assumptions. Total capacity is the sum of all three segments. Alpha at capacity is estimated through weighting by segment capacity. The critical factor determining 


\section{Charts of Model Outputs}

Panel A: Alpha and Capacity for Momentum Per Segment

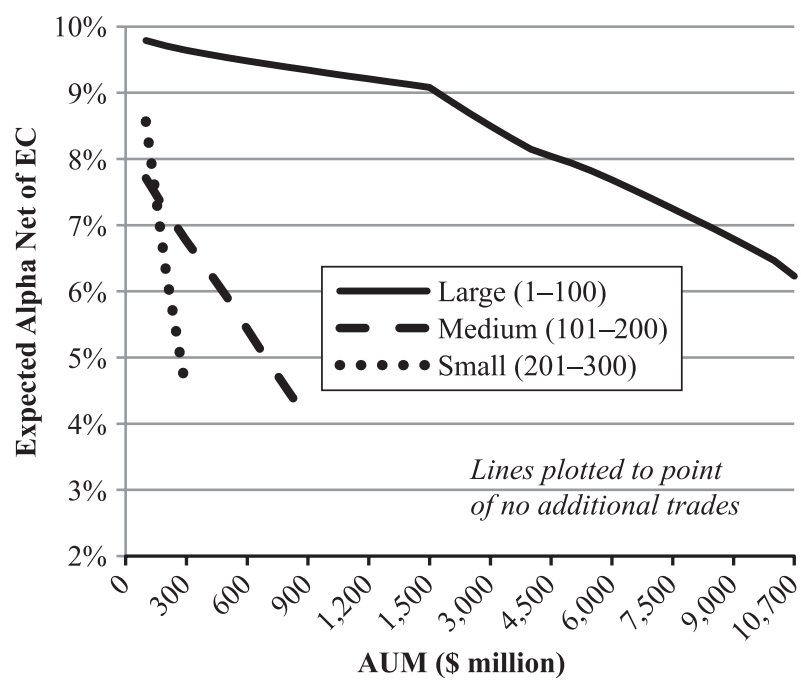

Panel C: Momentum Implementation Shortfall: Medium Size Segment

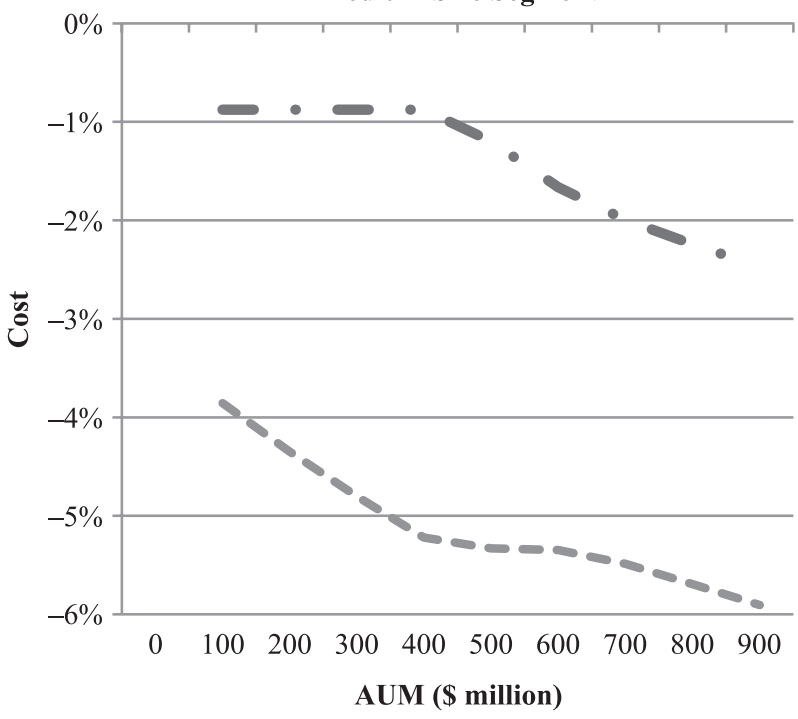

Panel B: Alpha and Capacity for IML Per Segment

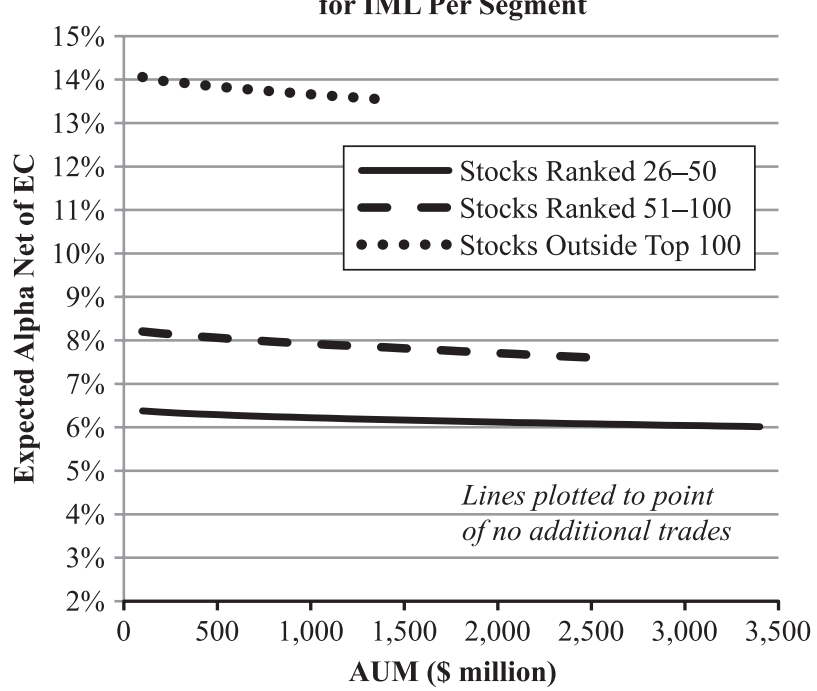

Panel D: IML Implementation Shortfall: Stocks Ranked 51-100

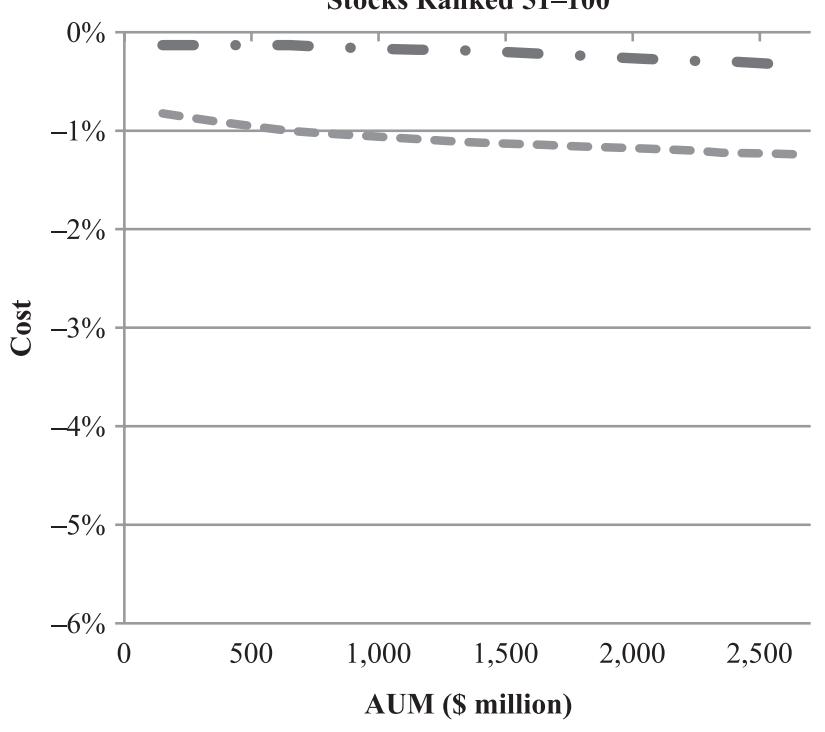

- Opportunity Cost $-\infty-$ Execution Cost

capacity is identified as either the holding constraint becoming binding (denoted Holding), further trades being unable to generate sufficient alpha (Trading), or net alpha declining to the threshold (Threshold). The results reported in Exhibit 5 not only give a sense of the range for capacity, they also illustrate how the input assumptions interact to generate the capacity estimates and provide an appreciation of how the key drivers depend on the nature of the strategy and its alpha profile in particular.

For IML, capacity is always determined by the holding constraint. Furthermore, capacity is sensitive 


\section{E X H I B I T 5}

\section{Capacity and Alpha Estimates}

\begin{tabular}{|c|c|c|c|c|c|c|c|c|}
\hline \multirow{2}{*}{$\begin{array}{l}\text { Strategy Estimates } \\
\text { Segment }\end{array}$} & \multicolumn{4}{|c|}{ Momentum Strategy } & \multicolumn{4}{|c|}{ IML Active Component } \\
\hline & $\begin{array}{c}\text { Large } \\
(1-100)\end{array}$ & $\begin{array}{l}\text { Medium } \\
(101-200)\end{array}$ & $\begin{array}{c}\text { Small } \\
(201-300)\end{array}$ & Total & $\begin{array}{l}\text { Stocks Ranked } \\
26-50\end{array}$ & $\begin{array}{l}\text { Stocks Ranked } \\
51-100\end{array}$ & $\begin{array}{l}\text { Stocks Outside } \\
\text { Top } 100\end{array}$ & $\begin{array}{l}\text { Total } \\
\text { Active }\end{array}$ \\
\hline \multicolumn{9}{|l|}{ Baseline } \\
\hline Capacity Estimate ( $\$$ A billion) & 10.7 & 0.9 & 0.3 & 11.9 & 3.4 & 2.6 & 1.4 & 7.4 \\
\hline $\begin{array}{l}\text { Reason Why Capacity } \\
\text { Reached }\end{array}$ & Holding & $\begin{array}{l}\text { Holding } \\
\text { \& Trading }\end{array}$ & Holding & & Holding & Holding & Holding & \\
\hline Implementation Shortfall & $-5.8 \%$ & $-8.6 \%$ & $-12.0 \%$ & $-7.7 \%$ & $-1.2 \%$ & $-2.0 \%$ & $-2.7 \%$ & $-3.9 \%$ \\
\hline \multicolumn{9}{|l|}{ Holding Constraint of $10 \%$} \\
\hline Capacity Estimate ( $\$$ A billion) & 16.0 & 0.9 & 0.3 & 17.2 & 6.9 & 5.2 & 2.7 & 14.8 \\
\hline Change from Baseline & 5.3 & 0.0 & 0.0 & \begin{tabular}{|l|}
5.3 \\
\end{tabular} & 3.5 & 2.6 & 1.3 & 7.4 \\
\hline Reason Why Capacity Reached & Trading & Trading & Trading & & Holding & Holding & Holding & \\
\hline Alpha at Capacity & $4.5 \%$ & $4.1 \%$ & $4.5 \%$ & $4.5 \%$ & $5.8 \%$ & $7.0 \%$ & $13.1 \%$ & $7.6 \%$ \\
\hline Reason Why Capacity Reached & Trading & Trading & Trading & & Holding & Holding & Holding & \\
\hline Alpha at Capacity & $6.6 \%$ & $2.5 \%$ & $4.0 \%$ & $6.2 \%$ & $6.0 \%$ & $7.2 \%$ & $13.3 \%$ & $7.8 \%$ \\
\hline Change from Baseline & $0.4 \%$ & $-1.6 \%$ & $-0.5 \%$ & $0.2 \%$ & $0.0 \%$ & $-0.3 \%$ & $-0.2 \%$ & $-0.1 \%$ \\
\hline \multicolumn{9}{|l|}{ Trade Constraint $45 \%$} \\
\hline Capacity Estimate (\$A billion) & 10.7 & 0.9 & 0.3 & 11.9 & 3.4 & 2.6 & 1.4 & 7.4 \\
\hline Change from Baseline & 0.0 & 0 & 0 & \begin{tabular}{|l|}
0.0 \\
\end{tabular} & 0.0 & 0.0 & 0.0 & 0.0 \\
\hline Reason Why Capacity Reached & Holding & Holding & Holding & & Holding & Holding & Holding & \\
\hline Alpha at Capacity & $6.6 \%$ & $4.7 \%$ & $5.4 \%$ & $6.4 \%$ & $6.0 \%$ & $7.6 \%$ & $13.5 \%$ & $8.0 \%$ \\
\hline Change from Baseline & $0.4 \%$ & $0.6 \%$ & $0.9 \%$ & $0.4 \%$ & $0.0 \%$ & $0.1 \%$ & $0.0 \%$ & $0.0 \%$ \\
\hline \multicolumn{9}{|l|}{ High Execution Costs } \\
\hline Capacity Estimate (\$A billion) & 6.1 & 0.3 & 0.1 & 6.5 & 3.4 & 2.6 & 1.4 & 7.4 \\
\hline Reason Why Capacity Reached & Threshold & Threshold & Threshold & & Holding & Holding & Holding & \\
\hline Alpha at Capacity & $2.0 \%$ & $2.0 \%$ & $2.0 \%$ & $2.0 \%$ & $2.5 \%$ & $3.2 \%$ & $5.9 \%$ & $3.4 \%$ \\
\hline Change from Baseline & $-4.2 \%$ & $-2.1 \%$ & $-2.5 \%$ & $-4.0 \%$ & $-3.5 \%$ & $-4.3 \%$ & $-7.6 \%$ & $-4.6 \%$ \\
\hline \multicolumn{9}{|l|}{ Second Best Opportunities Used } \\
\hline Capacity Estimate ( $\$$ A billion) & 12.0 & 1.1 & 0.4 & 13.5 & n.a. & n.a. & n.a. & \\
\hline Change from Baseline & 1.3 & 0.2 & 0.1 & 1.6 & & & & \\
\hline Reason Why Capacity Reached & Trading & Trading & Trading & & & & & \\
\hline Alpha at Capacity & $5.6 \%$ & $3.4 \%$ & $3.6 \%$ & $5.3 \%$ & & & & \\
\hline Change from Baseline & $-0.6 \%$ & $-0.7 \%$ & $-0.9 \%$ & $-0.7 \%$ & & & & \\
\hline \multicolumn{9}{|l|}{ Number of Positions Halved } \\
\hline Capacity Estimate (\$A billion) & 5.4 & 0.5 & 0.2 & 6.0 & 1.7 & 1.3 & 0.7 & 3.7 \\
\hline Change from Baseline & -5.4 & -0.5 & -0.2 & -6.0 & -1.7 & -1.3 & -0.7 & -3.7 \\
\hline Reason Why Capacity Reached & Holding & Holding & Holding & & Holding & Holding & Holding & \\
\hline Alpha at Capacity & $6.2 \%$ & $4.1 \%$ & $4.5 \%$ & $6.0 \%$ & $6.0 \%$ & $7.5 \%$ & $13.5 \%$ & $7.9 \%$ \\
\hline
\end{tabular}

Holding = holding constraint binding; Trading = further trades possible, but generate insufficient alpha; Threshold = 2\% alpha threshold reached . 
only to the holding constraint and the assumed number of positions, with estimates being directly affected by a change in either of these variables. All other variables impact only on expected alpha. These findings flow from an alpha profile that creates little urgency to trade, thus rendering assumptions related to trading and EC largely irrelevant for capacity. This indicates that capacity for this fund is determined largely by the number of stock opportunities it is able to identify and the market segments from which these opportunities are sourced.

For the momentum strategy, as the assumptions are varied, the critical factor determining capacity switches among the holding constraint, inability to invest further AUM at adequate alpha, and erosion of alpha to the threshold, although the latter only becomes binding if alpha is reduced by $50 \%$. Changes to assumptions related to trading - notably a reduced trade participation constraint and increased EC using high-end parameter inputs based on history - act to reduce capacity as well as alpha. This occurs because it becomes more difficult to capture alpha opportunities by trading quickly or at an acceptable cost. Access to opportunities also affects capacity for the momentum strategy. This is revealed by a halving of capacity when the number of stock holdings is reduced by $50 \%$ and increases in capacity when secondbest opportunities are accessed. The latter is accompanied by a reduction in alpha, as the additional AUM is invested at a diminished level of return. Overall, the potential determinants of capacity for the momentum strategy are broader and more nuanced. An approach that integrates all drivers can be useful in such situations because it allows the potential pressure points to emerge.

Finally, this analysis represents the active alphaseeking component of a portfolio. IML manages a family of long-only funds reflecting a combination of alpha and beta. Blending the baseline capacity estimate for the IML alpha component of $\mathrm{A} \$ 7.4$ billion with a $50 \%$ core or beta component points to capacity of $\mathrm{A} \$ 14.8$ billion for the IML long-only equity funds based around this strategy and portfolio structure. Another challenge is relating the capacity estimate to all funds managed by IML. Our analysis is based on the flagship fund, which had AUM of A $\$ 2.3$ billion in June 2016, out of a total AUM of $\$ 6.0$ billion. We estimate $89 \%$ overlap in stocks held in the flagship fund across all funds in the stable. Grossing up for this partial overlap suggests that capacity for the IML fund group is in the order of
$\mathrm{A} \$ 16.6$ billion, assuming that nonoverlapping positions can be similarly scaled.

\section{CONCLUSION}

This article aims to demonstrate how the analysis of capacity for active equity funds may be undertaken. We discuss the key influences and how they determine capacity across differing strategies. We propose an approach that combines the key drivers in an integrated analysis and illustrate the approach using data for a quantitative momentum strategy and a stock-picking equity fund. A key message is that capacity analysis should focus on the point at which additional AUM is unable to be actively invested at a marginal alpha exceeding some minimum threshold-a quantity that we call effective capacity - rather than focusing on total fund alpha. A fund may be generating substantial alpha from the opportunities in which it has already invested, yet is unable to generate sufficient marginal alpha on further AUM. This situation may occur as a consequence of holding or trading constraints restricting the amount or pace of investment, suggesting that it is crucial to address such constraints when conducting capacity analysis. Furthermore, capacity may be determined by a range of drivers, which in turn may interact and can differ in importance depending on the strategy and its alpha profile. We contend that the most effective way to account for the multiple potential influences is to apply an integrated approach of the type presented in this article.

\section{ENDNOTES}

${ }^{1}$ We use the term alpha in a generic sense to represent the active return in excess of that required by the investor. This encapsulates various benchmarks for required return, including an index return, required return adjusting for factor or risk exposure, or perhaps a target absolute return.

${ }^{2}$ For instance, Kahn and Shaffer [2005]; Serbin, Bull, and Zhu [2009]; and Coppejans and Madhavan [2007] developed their models in a single-period mean-variance framework aligning with the fundamental law of active management of Grinold [1989], where alpha is assumed to decay at a constant rate per period and turnover provides the key lever to control transaction costs.

${ }^{3}$ An exception is Indro et al. [1999], who regressed fund performance against size to estimate the optimal AUM for three fund styles: value, blend, and growth. 
discussion.

${ }^{4}$ Refer to O'Neill and Warren [2016] for a more detailed

${ }^{5}$ Vangelisti [2006] also refers to terminal capacity, which is the funds under management at which net alpha is reduced to zero. We discard this as a working definition because it assumes no residual benefit for investors, even though it may maximize revenue for the fund management company.

${ }^{6}$ The alpha index should reflect cumulative relative wealth over time. For example, if alpha were defined as excess return versus an index benchmark, then an alpha index series can be created as $C A_{S, t+x}=\left(S I_{S, t+x} / S I_{s, t}\right) /\left(B I_{t+x} / B I_{t}\right)$, where $C A$ is the cumulative alpha index, $S I$ is an accumulation index for stock $s, B I$ is the accumulation index for the benchmark index, $t$ is the decision point, and $x$ is the estimation time period. Alpha indexes might be generated on a daily basis and converted to weekly or monthly observations for the purpose of analysis as appropriate.

${ }^{7}$ Turnover for the active component will exceed total portfolio turnover if there is a beta component.

${ }^{8}$ The industry and many transaction cost models often refer to average daily volume. Because capacity is being modeled in dollar values and the analysis could be based around intervals other than a day, the average value of trades per unit of time becomes the relevant reference point.

${ }^{9}$ Refer to O'Neill and Warren [2016] for discussion of the other factors and drivers.

${ }^{10}$ The estimates are indicative rather than precise. Also, the scales on the horizontal axes are not linear, making it important to reference the AUM numbers on the $x$-axis directly.

\section{REFERENCES}

Almgren, R., and N. Chriss. "Optimal Execution of Portfolio Transactions." Journal of Risk, 3 (2001), pp. 5-40.

Almgren, R., C. Thum, E. Hauptmann, and H. Li. "Direct Estimation of Equity Market Impact." Risk, Vol. 18, No. 7 (2005), pp. 58-62.

Amihud, Y., and H. Mendelson. "Transaction Costs and Asset Management." In Global Asset Management, edited by M. Pinedo and I. Walter, pp. 414-432. Basingstoke, UK: Palgrave Macmillan, 2013.

Arnott, R.D., and W.H. Wagner. "The Measurement and Control of Trading Costs." Financial Analysts Journal, Vol. 46, No. 6 (1990), pp. 73-90.

Bertsimas, D., and A.W. Lo. "Optimal Control of Execution Costs." Journal of Financial Markets, Vol. 1, No. 1 (1998), pp. $1-50$.
Chen, Z., W. Stanzl, and M. Watanabe. "Price Impact Costs and the Limit of Arbitrage." Working paper, Yale School of Management, December 7, 2005.

Coppejans, M., and A. Madhavan. "The Value of Transaction Cost Forecasts: Another Source of Alpha." The Journal of Investment Management, Vol. 5, No. 1 (2007), pp. 65-78.

Cremers, K.J.M., and A. Petajisto. "How Active Is Your Fund Manager? A New Measure That Predicts Performance." Review of Financial Studies, Vol. 22, No. 9 (2009), pp. 3329-3365.

Engle, R.F., and R. Ferstenberg. "Execution Risk." The Journal of Portfolio Management, Vol. 33, No. 2 (2007), pp. 34-44.

Frazzini, A., R. Israel, and T. Moskowitz. "Trading Costs of Asset Pricing Anomalies." Working paper no. 14-05, FamaMiller, Chicago Booth School of Business, 2012.

Gârleanu, N., and L.H. Pedersen. "Dynamic Trading with Predictable Returns and Transaction Costs." Journal of Finance, Vol. 68, No. 6 (2013), pp. 2309-2340.

Grinold, R.C. "The Fundamental Law of Active Management." The Journal of Portfolio Management, Vol. 15, No. 3 (1989), pp. 30-37.

Huberman, G., and W. Stanzl. "Optimal Liquidity Trading." Review of Finance, Vol. 9, No. 2 (2005), pp. 165-200.

Indro, D.C., C.X. Jiang, M.Y. Hu, and W.Y. Lee. "Mutual Fund Performance: Does Fund Size Matter?" Financial Analysts Journal, Vol. 55, No. 3 (1999), pp. 74-87.

Kahn, R.N., and J.S. Shaffer. "The Surprisingly Small Impact of Asset Growth on Expected Alpha." The Journal of Portfolio Management, Vol. 32, No. 1 (2005), pp. 49-60.

Korajczyk, R.A., and R. Sadka. "Are Momentum Profits Robust to Trading Costs?" The Journal of Finance, Vol. 59, No. 3 (2004), pp. 1039-1082.

Landier, A., G. Simon, and D. Thesmar. "The Capacity of Trading Strategies.” Research paper No. FIN-2015-1089, HEC Paris, 2015.

Novy-Marx, R., and M. Velikov. "A Taxonomy of Anomalies and Their Trading Costs." Review of Financial Studies, Vol. 29, No. 1 (2016), pp. 104-147. 
O’Neill, M., C. Schmidt, and G. Warren. "Capacity Analysis." Working paper, No. 129/2016, CIFR Research, December 2016.

O’Neill, M., and G. Warren. "Evaluating Fund Capacity: Issues and Methods." Working paper, No. 124/2016, CIFR Research, September 2016.

Perold, A.F. "The Implementation Shortfall: Paper versus Reality." The Journal of Portfolio Management, Vol. 14, No. 3 (1988), pp. 4-9.

Perold, A.F., and R.S. Salomon Jr. "The Right Amount of Assets under Management." Financial Analysts Journal, Vol. 47, No. 3 (1991), pp. 31-39.

Serbin, V., P.M. Bull, and H. Zhu. "The Capacity of Liquidity-Demanding Equity Strategies.” The Journal of Portfolio Management, Vol. 36, No. 1 (2009), pp. 78-89.

Vangelisti, M. "The Capacity of an Equity Strategy." The Journal of Portfolio Management, Vol. 32, No. 2 (2006), pp. 44-50.

To order reprints of this article, please contact David Rowe at drowe@iijournals.com or 212-224-3045. 
Reproduced with permission of copyright owner. Further reproduction prohibited without permission. 